\title{
BANQUES CAMEROUNAISES ET «DISCOURS RELATIONNEL" SUR FACEBOOK: SOCIOSÉMIOTIQUE DES INSTANCES ET MODALITÉS DE PERFORMANCE
}

\section{CAMEROONIAN BANKS AND "RELATIONAL DISCOURSE" ON FACEBOOK: A SOCIO-SEMIOTICS OF INSTANCES AND PERFORMANCE MODALITIES}

LÉOPOLD NGODJI TCHEUTOU* ALPHONSE JOSEPH TONYE ${ }^{* *}$

RÉSUMÉ: Analyser les instances et les modalités de performance de deux banques camerounaises (SCB Cameroun et Société Générale Cameroun) à travers leur « discours relationnel » sur Facebook peut s'avérer porteur de sens dans le secteur bancaire. Par le biais d'un échange dynamique, caractérisé par l'interaction, nous procédons par une approche sociosémiotique pour questionner le rôle du «marketing relationnel $2.0 »$ dans la performance des marques bancaires en contexte. L'on note qu'à côté du faible taux

\footnotetext{
* Doctorant à l’UYI - Université de Yaoundé I. Email: leopoldngodji@yahoo.fr.

** Professeur à l’UYI - Université de Yaoundé I. Email: ajtonyecm@yahoo.fr.
} 
d'« engagement » des pages facebook des marques bancaires camerounaises, leur discours relationnel sur Facebook rentre en permanence en conflit avec ceux des internautes sous la forme de désaccord verbal, inaperçu et apparemment mal géré par les communities managers des pages en question. Ce conflit rend ainsi imperceptible le rôle majeur que pourrait jouer cette plateforme, à côté des autres instances dans la performance des banques dans leurs quêtes de recrutement et de rétention des clients d'une part, d'association et de partage des valeurs et projets de l'entreprise par les ciblesinternautes, d'autre part.

MOTS-CLÉS: Instances. Modalités. Discours relationnel. Marketing relationnel 2.0. Conflit. Performance. Sociosémiotique.

ABSTRACT: An analysis of the instances and the performance modalities of two Cameroonian banks (SCB Cameroon and Société Générale Cameroon) through their "relational discourse" on Facebook might be meaningful for the banking sector. Under some conditions of interactive exchange, determined by interaction, we proceed by a socio-semiotic approach to question the role of "relational Marketing 2.0" over the bank's brand performance in context. In addition, we found that besides the low rate of "commitment" observed on facebook pages of Cameroonian banking brands, their relational discourse on Facebook post is constantly in conflict with those of Internet users in the form of verbal disagreement, unnoticed and apparently mismanaged by the communities managers of those pages. This conflict also makes imperceptible the major role that could play a social media like Facebook. On the one hand, alongside other 
instances in the performance of banks within their objective of customers' recruitment and retention, and on the other hand the association and the sharing of values and projects of the enterprise by Facebook-targets.

KEYWORDS: Instances. Modalities. Relational discourse. Relational marketing 2.0. Conflict. Performance. Sociosemiotics.

\section{En guise d'introduction}

Grâce à l'évolution géométrique des TIC (Technologies de l'information et de la communication) dans nos sociétés, les habitudes ainsi que les formes d'expressions socioculturelles se sont modelées depuis plus d'une décennie. Concomitamment, les pratiques professionnelles dans un certain nombre de secteurs économique, industriel et commercial se sont arrimées à ces mutations post-modernes.

Les établissements de crédit, notamment dans leur considération technique de marques bancaires, constituent aujourd'hui, auxyeux de plusieurs spécialistes, un des secteurs par excellence de la transformation observée: réseaux bancaires consolidés, transactions financières fortement informatisées, payement par carte magnétique, nouveaux métiers de la banque, bref, toutes les activités autour de ce qu'il convient d'appeler l'E.banking. Pour reprendre les propos de Serres (2012), la révolution numérique en cours aura des effets au moins aussi considérables qu'en leur temps l'invention de l'écriture puis celle de l'imprimerie.

Si les métiers et les pratiques bancaires se sont transformés pour converger vers la «banque digitale», la 
relation client se positionne comme l'un des axes stratégiques sur lesquels le marketing bancaire 2.0 joue un rôle essentiel. À partir d'un ordinateur ou d'un Ipad et Ipod, ou encore d'un téléphone androïd (même ordinaire), l'on peut désormais consulter son compte en ligne, y faire des achats, recevoir des alertes sous la forme d'e.mail et/ou de SMS (short message service), échanger en temps réel par téléphone ou sur les médias sociaux (numériques) avec son gestionnaire de compte ou un community manager des médias sociaux. Concernant l'intéressement stratégique aux réseaux sociaux principalement (Twitter, YouTube, facebook...), nombreuses sont les banques qui s'y sont accommodées malgré les réticences et les idées reçues qui ont ralenti l'appropriation de l'outil.

Comment les marques bancaires construisent-elles leurs discours relationnels sur les médias sociaux, en l'occurrence sur Facebook ? En d'autres termes, de quelle manière, avec quelle importance et comment le marketing relationnel 2.0, notamment les prises de parole des marques bancaires sur Facebook, peut-il contribuer à la performance globale des établissements de crédit ? Cette question nous permet de décrypter les prises en charge des discours relationnels de deux banques de détail camerounaises (SCB Cameroun et Société Générale Cameroun) sur Facebook. Pour ce faire, nous avons choisi deux thématiques abordées par ces dernières à l'effet de discuter sur les instances et les modalités propositionnelles à partir desquelles elles s'expriment et rentrent dans un dispositif d'interaction avec leurs internautes respectifs.

Il faut dire que sur le plan technique, les lignes de convergence discursive et de désaccord verbal dans les posts entre les sujets interactifs ou co-énonciateurs sont 
analysées en vue de décrypter les forces et les faiblesses des deux banques grâce aux variables liées à la confiance, la proximité, l'engagement, l'interactivité, l'instantanéité etc. Le désaccord verbal étant à considérer comme «une forme de conflit, parce que les désaccords verbaux mettent à l'épreuve les occurrences de communication, caractérisées par des buts incompatibles, de la négociation et la nécessité de coordonner ses propres actions avec celles des autres», (WALDRON; APPLEGATE, 1994, p. 4, notre traduction) ${ }^{1}$.

Pour conduire notre analyse, nous avons mobilisé les approches sociosémiotiques de Landowski et Semprini. Cette théorie nous a permis de prendre en compte l'idée d'une interaction entre les acteurs de l'échange en termes de transformation et de circulation d'un «énoncé énoncé» tel que le propose Éric Landowski dans son ouvrage La société réfléchie, encore d'actualité :

[...] au lieu d'envisager le langage comme le simple support de «messages» circulant entre des émetteurs et des récepteurs quelconques [...], il s'agira avant tout de saisir les interactions réalisées, à l'aide du discours, entre les «sujets», individuels ou collectifs, qui s'y inscrivent et qui, d'une certaine façon, s'y reconnaissent. Landowski (1989, p. 8-9).

Le regard sociosémiotique développé par Andrea Semprini nous aide à mieux comprendre la portée du sens qui naît dans un énoncé lors de sa «rencontre» avec le dispositif social qu'est la communauté facebook en termes de prise en charge des discours:

1 Selon Waldron et Applegate, le désaccord verbal peut s'appréhender comme «a form of conflict, because verbal disagreements are taxing communication events, characterized by incompatible goals, negociation, and the need to coordinate self and others actions». 
Dans l'analyse d'un énoncé déterminé, il s'agit en plus naturellement de l'analyse de l'énoncé lui-même, qui demeure un point fixe et distinctif de toute approche sémiotique, de questionner la légitimité ou la possibilité même pour l'énoncé de se transformer en discours et de circuler dans l'espace social et dans la sémiosphère (SEMPRINI, 2007, p. 17).

Ainsi, le questionnement de la prise en charge des posts des deux banques sur leur page Facebook est de natureà mettre en valeur le fonctionnement signifiant qui devrait permettre à ces deux banques de projeter leurs performances respectives. La saisie du marketing relationnel 2.0 des établissements de crédit (1) et la prise en charge du discours relationnel des deux banques (2) constituent les deux ancrages de ce travail.

\section{De la saisie du marketing relationnel 2.0 des établissements de crédit}

L'activité bancaire est essentiellement une activitérisque. De tradition pour les établissements de crédit, autrement appelés les banques, et précisément le cas des marques bancaires, la qualité du service a toujours été une préoccupation fondamentale dans les logiques de performance qui les caractérisent. Ces enjeux, sous la forme de contraintes et de gouvernance bancaire sont le fait, le plus souvent, des organismes de régulation et de contrôle d'essence prudentielle. Pour ce faire, si longtemps, la performance des institutions bancaires s'est «pesée» sous des «balances» de type quantitatif et comptable, des études relativement récentes reconnaissent la place des indicateurs non-comptables et qualitatifs l'évaluation des entreprises bancaires. 
D'autres perspectives comme l'approche du marketing relationnel existent et permettent d'appréhender la performance des institutions bancaires. En effet, parler de la performance dans sa seule dimension économique, financière et comptable ne permet pas toujours de cerner toutes les variables intangibles et immatérielles de ce concept (PESQUIEU, 2004). Ainsi, l'approche relationnelle d'une banque et plus précisément son «discours relationnel», apparaît comme l'un des lieux privilégiés de perception de la quête de l'efficience et de l'efficacité des institutions bancaires, bref, de la dimension qualitative, non-financière et non-comptable.

Nous partons de la considération selon laquelle «pour le financier, la marque est la confiance. Elle est la réputation» (KAPFERER, 2005, p. 27). C'est donc dire que la confiance est l'un des déterminants caractéristiques des marques bancaires qu'il convient de mettre en valeur dans un contexte de concurrence: «une marque bancaire est aussi un capital. Elle satisfait en effet trois critères importants en termes de valorisation : la notoriété, l'attractivité et la fidélité à la marque» (BOTTON, 2005, p. 34).

À proprement parler, c'est dans cette optique qu'il faut considérer la prise de parole des marques sur leurs pages Facebook respectives en termes de parole tenue suivant une approche relationnelle qui vise le long terme, l'interactivité, la considération du client-partenaire, etc. Comme on peut l'observer,l'approche relationnelle estdevenue prépondérante dans l'échange commercial que la banque entretient au quotidien lors de ses «rencontres» avec ses clients et prospects dans un triple objectif: recrutement, consolidation du lien et rétention du client. Cette approche relationnelle, faut-il le rappeler, privilégie un échange centré sur la qualité de service 
et le client vers qui la banque construit et promeut les valeurs de partage, de communication, d'interactivité, de proximité, de sincérité:

L'engagement et la confiance étaient toujours théorisés comme des variables centrales du modèle du marketing relationnel avec un nombre de facteurs l'accompagnant (coût, communication, partage des valeurs, etc.) avec des issus (conflit, propension de quitter la relation, coopération, etc.) par chaque partie prenante. (...) Toutefois, des études évaluatrices ont montré des liens communs sur les recherches relatives à ces deux valeurs de manière explicite ou implicite. Ces concepts communs sont la valeur, les effets de long terme, l'interaction, la coopération, le travail en réseau, l'utilisation efficiente des ressources de l'entreprise, la confiance et l'engagement. (GANGULI; ESHGHI; BECHWATI, 2009, p. 3-6, notre traduction) $)^{2}$.

À travers ces précisions sur les concepts de performance, de marketing relationnel et de marques bancaires, l'on peut mieux comprendre pourquoi les grandes préoccupations discursives des banques en direction de leurs clients se campent sur la qualité de service à construire et à mettre en valeur tant sur le plan matériel que sur le plan purement imaginaire et intangible.

Sur le plan purement marketing, l'on peut bien se demander ce que viendraient chercher les concepts d'instance et de modalité dans le secteur bancaire où les techniques de

2 Commitment and trust were also theorized to be the key mediating variables in a relationship marketing model with a number of precursors (costs, communication, shared values etc.) and outcomes (conflict, propensity to exit a relationship, cooperation etc.) for each. [...] However, a critical evaluation reveals certain common concepts among the research streams either explicitly or implicitly. These common concepts are value, long term effects, interaction, cooperation, networking, resource utilization, trust and commitment. (GANGULI; ESHGHI; BECHWATI, 2009, p. 3-6). 
mesure de performance sont prédominées par les tableaux de bord et les autres instruments de type comptable. C'est parce qu'avec les travaux comme ceux de Morin, Savoie et Beaudin (1994), il est reconnu qu'aujourd'hui les indicateurs financiers et comptables de la performance des banques ne sont que la partie finie d'un ensemble de variables d'abord opérationnelles et très souvent non-quantifiables telles que la légitimité de l'entreprise à travers son approche relationnelle, la politique de pérennité de l'entreprise reconnue par les clients, la valorisation des personnels par les clients et une recherche collective de l'efficience économique.

À tout prendre dans leur signification, ces indicateurs qualitatifs et non-financiers peuvent se matérialiser et se percevoir à partir des «lieux» et des «conditions» qui permettent de valider la portée de la rencontre du «discours relationnel» et de l'échange commercial entre les parties prenantes de l'activité bancaire.

Dans ce prolongement, l'on dira que le concept d'instance, du latin instantia, signifierait «imminence, proximité, présence». Parler d'instance dans un phénomène revient donc à questionner et à identifier les différents niveaux pertinents, mieux les points de proximité à partir desquels, le sens naît. Autrement dit, les instances [on peut distinguer les autres instances de la rencontre relationnelle que sont les «espaces proxémiques de la relation» (à savoir les occasions de rencontre directe du personnel bancaire avec la clientèle) et les «espaces graphiques de la relation» (tous les supports et moyens de communication commerciale, institutionnelle et publicitaire que la banque produit en direction de sa clientèle)], notamment les «espaces numériques de la relation bancaire» constituent des lieux signifiants à travers lesquels la marque échange avec sa «clientèle-digitale». 
Quant à la modalité, elle revient dans la subjectivité énonciative du sujet parlant (modalités d'énonciation et modalités propositionnelles). Le terme modalité se définit alors comme une attitude réactive du sujet énonçant vis-àvis d'un contenu discursif (GOSSELIN, 2001, p. 45). Le terme comporte un «[...] sujet modal [qui] peut être et est le plus souvent en même temps le sujet parlant» (BALLY, 1965, p. 37). Dans la sémiotique narrative, elle renvoie aux conditions qui permettent à un «sujet» d'être en relation avec son « objet de valeur» en termes de transformation. Jacques Fontanille (1998, p. 140) évoque la notion avec précision:

Les modalités sont des contenus qui définissent l'identité des actants. [...] [La modalité] [...] porte aussi sur les actants, en ce sens que le contenu sémantique de la modalité peut être considéré comme une propriété de l'actant lui-même, propriété nécessaire pour qu'il réalise l'acte.

Danslecontextebancaire, etcesurun rapportdesignifiant et d'expression énonciative, les instances et les modalités constituent des points d'achoppement de production et de circulation à travers lesquels le «discours relationnel» prend corps. Le terme discours étant à prendre ici comme l'ensemble des productions textuelles icono-plastiques et relationnelles qui lient toutes les parties prenantes de l'activité bancaire au quotidien, à savoir: le personnel bancaire, les prospects et clients, les partenaires institutionnels et réglementaires, les actionnaires et les fournisseurs, les facebookers. En somme, tous ceux ou celles qui entretiennent une relation avec la banque sous quelque rapport que ce soit.

Qu'est-ce qui aurait changé entre temps dans la relation banque-client? Parmi les esquisses de réponses possibles ayant révolutionné les pratiques bancaires, les technologies 
de l'information et de la communication, à savoir Internet et le Web 2.0 y occupent une place prépondérante au cours de ces dernières années.

Il faut dire que l'étiquette 2.0, que l'on accole aujourd'hui à tous les domaines (communication 2.0, culture 2.0, politique 2.0, stratégies 2.0, entreprise 2.0, marketing 2.0, banque 2.0, etc.) désigne l'ensemble des pratiques et des usages d'Internet et qui privilégie la participation, la contribution active des individus et une multiplication des échanges. (REBILLARD, 2011). Les médias sociaux constituent alors la dernière forme des applications basées sur le 2.0 et désignent une série d'innovations technologiques en termes de hardware et software qui facilitent la création de contenus à faible coût, l'interaction et l'interopérabilité par les utilisateurs en ligne (BERTHON et al., 2012).

Dans la même logique, le rapide succès des réseaux sociaux dans toutes les sphères de la vie sociale, publique et professionnelle n'est plus à démontrer (BALAGUÉ; FAYON, 2012, p. 6):

Pour toucher 50 millions d'utilisateurs, il a fallu trente-huit ans à la radio, treize ans à la télévision, quatre ans pour Internet, trois ans à l'iPod... tandis que Google+ a conquis 50 millions de membres en 88 jours, soit la période de révolution de Mercure! Plus de 300 millions de photos sont téléchargées quotidiennement sur Facebook et 3,2 milliards de J'aime et de commentaires effectués par les utilisateurs! Chaque jour, de nouveaux réseaux sociaux, dédiés à des usages spécifiques ou à des cibles différenciées, apparaissent et s'intègrent à nos vies.

Comme on peut le constater, ces données statistiques liées à l'évolution du Web 2.0 plaident en faveur de ce succès qui contraint toutes les formes d'organisations et d'entreprises 
sans restriction aucune à s'intéresser sinon à s'intégrer dans la communauté 2.0.

Par une vitesse relativement lente mais significativement perceptible au fil du temps, les marques bancaires camerounaises s'intéressent, s'intègrent et s'insèrent progressivement dans ce nouvel univers. Pour construire la confiance et développer des échanges relationnels de forme modale multidimensionnelle (le faire-connaître, le fairesavoir, le faire-partager, le faire-faire, etc.), quelques banques camerounaises ont pris du temps pour observer et prendre la mesure de l'inscription de leur prise de parole en tant que sujet énonçant sur leur page Facebook en l'occurrence. C'est le cas de SCB Cameroun et Société Générale Cameroun, objet des présents développements.

\section{Facebook et prise en charge du discours relationnel de SCB Cameroun et Société Générale Cameroun}

En guise de rappel, les posts de notre étude portent respectivement sur les photos de couverture figurant sur les pages Facebook de SCB Cameroun (Image 1) et de Société Générale Cameroun (Image 3) lors de leur capture en date de 05 septembre 2015; et les annonces d'ouverture de nouvelles agences de SCB Cameroun (Image 2) et de Société Générale Cameroun (Image 4). Les dates de mise en ligne des quatre posts en question sont respectivement le 26 mai 2015 (22:46), le 17 avril 2015 (19:45), le 22 décembre 2014 (23:30) et le 19 mai 2015 (12:31).

Il està noter quele plus grand nombre de «commentaires» des posts (Image 4) est de 15, et le plus grand nombre de «j'aime» (Image 1) est de 30 à la date du 05 septembre 2015. 
À l'observation, ces «taux d'engagement» sont encore très faibles au vu des opportunités et des possibilités qu'offre la plateforme Facebook aujourd'hui dans un pays d'environ 25 millions d'habitants avec au premier trimestre 2015 un peu plus de 1.100 .000 facebookers dont 700.000 hommes et 400.000 femmes (MINISTÈRE EN CHARGE DES TELECOMMUNICATIONS, 2015).

\subsection{Posts de SCB Cameroun: de l'ontique à l'épistémique}

Sur le premier post de la SCB Cameroun (Image 1), l'on a affaire à une prise de parole descriptive qui résume l'activité de la banque. On y lit: «SCB Cameroun. Banque des particuliers. Banque de financement. Banque des marchés». Ce texte est suivi des figures polygonales et multicolores en montage 3D sur lesquelles sont inscrites en détails les services qui composent les activités de l'entreprise: «Pack SCB, Cartes bancaires, Marchés de change, E.bank, Créditbail, Financement, Placements, Assurances, découvertes, International, cash express» etc. Le tout est clôturé par une création infographique, en forme de lignes brisées de trois pieds, qui laisse en filigrane une salle de conférence bien meublée. Puis le logo de la banque en baseline. En fait, ces lignes brisées ne sont qu'une représentation allusive du logo de la banque SCB Cameroun, filiale du groupe bancaire marocain Attijari Wafa au Cameroun. Toutes ces images iconiques sont «soutenues» par un fond plastique de couleur rouge jaunâtre en adéquation avec la charte graphique et visuelle de la banque. 
Image 1 - Capture d'écran du post de la photo de couverture de la page facebook de SCB Cameroun

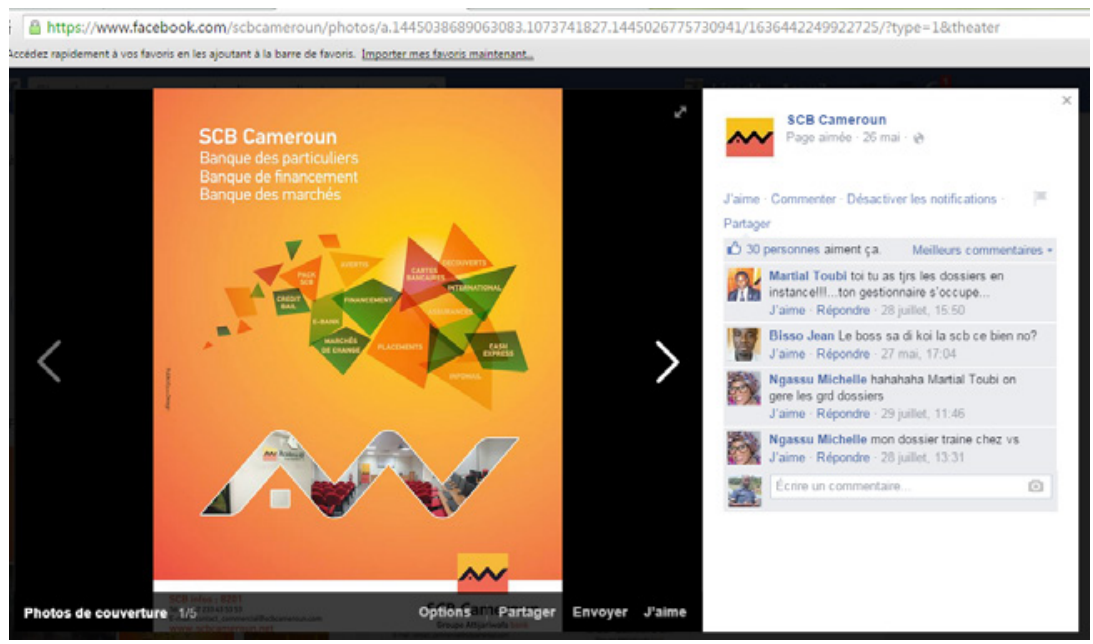

Source: Page Facebook de SCB Cameroun

Image 2 - Capture d'écran du post d'écran relatif à l'ouverture d'une agence SCB Cameroun

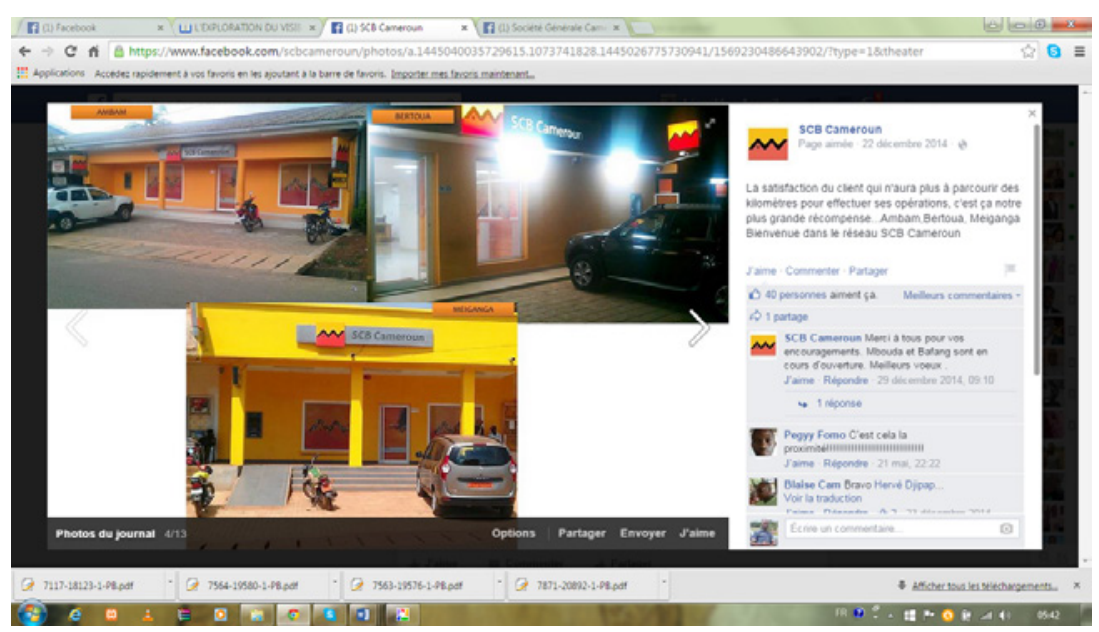

Source: Page Facebook de SCB Cameroun 
Comme on peut l'observer sur la page Facebook de la banque, c'est certainement parce que le post est mis en ligne pour servir de photo de couverture de la page qu'il n'est accompagné par aucun texte. En principe, une photo de couverture d'une page Facebook est une photo marquante choisie pour servir d'identité à la page suivant une durée. Cette photo peut avoir à la fois une fonction d'identité et de mémorisation d'une campagne ou d'un événement majeur de l'entreprise. Pour le cas en question, il s'agit d'une image qui présente en détail les trois activités principales de SCB Cameroun, à savoir: (1) des services aux particuliers, (2) des services de financement de l'économie et (3) des services des marchés hypothèques.

En tant que sujet énonçant (et co-énonciateur en pratique), à savoir l'être de parole (ou d'énonciation) qui est construit par l'acte d'énonciation du sujet communicant inscrit dans la mise en scène du dire, (CHARAUDEAU; MAINGUENEAU, 2002), SCB Cameroun prend la parole ici de manière implicite pour mettre en discussion un propos relationnel d'exposition et de description de ses activités sous la forme de résumé modal suivant : «je suis ...» et «j'assure à la fois les services suivants...». Concrètement, on peut dire qu'il s'agit d'un discours relationnel qui affirme une modalité ontique (l'être) vers des modalités épistémiques (le certain). D'où l'affirmation de l'individualisme de la banque de l'ontique à l'épistémique.

Le deuxième post de la SCB Cameroun (Image 2) est un message d'annonce de l'ouverture de trois nouvelles agences dans les villes de Bertoua, Ambam et Meiganga, situées respectivement dans les régions Est, Sud et Nord du Cameroun. On y voit trois prises de photo présentant les façades des immeubles abritant les agences nouvellement ouvertes aux 
couleurs de l'entreprise (rouge et jaunâtre). Ces façades principales qui portent des plaques d'indentification de la banque sont occupées par des véhicules et des motocyclettes.

À première vue, le choix de poster des photos réelles se justifie par la volonté de l'entreprise de montrer le confort et les commodités spatio-topographiques pour les clients de l'agence, et ainsi affirmer que d'ores et déjà, ces trois agences sont ouvertes et fonctionnelles.

Sur la page, on lit au dessus de l'image un message qui accompagne ces prises de photo: «La satisfaction du client qui n'aura plus à parcourir des kilomètres pour effectuer ses opérations, c'est ça notre plus grande récompense... Ambam, Bertoua, Meiganga, Bienvenue dans le réseau SCB Cameroun».

Ce texte met en valeur deux caractéristiques essentiellement de la relation banque-clients, à savoir: la satisfaction et la proximité. Sur le plan constructif, l'énonciateur SCB Cameroun s'inscrit ainsi dans un discours auto-glorifiant et personnifiant en direction des clients, bénéficiaires finaux de l'activité bancaire.

En présentant l'ouverture de ces agences comme une «récompense», soutenue par un «nous» exclusif et individuel, «c'est ça notre...», qui annonce la «bienvenue dans le réseau», mieux la bienvenue dans l'«univers» de la banque sous-entend une fois de plus un individualisme de son discours relationnel qui peut se traduire par les expressions suivantes : «je suis ...» et «j'assure la satisfaction... Je récompense...». En gros, comme dans le cas du post relatif à la photo de couverture, l'affirmation d'une modalité ontique (l'être) vers des modalités épistémiques (le certain et le plausible) est encore bien perceptible.

Sur un tout autre plan, cette manifestation de l'individualisme de SCB Cameroun à travers un discours 
relationnel qui va de l'ontique vers l'épistémique peut être appréhendée sous le prisme mythologique, c'est-à-dire un ensemble de mythes, de croyances et d'idéologies qui s'organise autour d'un thème de manière explicite ou implicite.

Comme dans tous les mythes des marques, c'est donc l'énonciateur SCB Cameroun qui se positionne comme un héros épique salvateur qui glorifie et qui récompense à travers trois des sept «cartes mentales» de Lewi, (2009): (1) le matériel et l'immatériel (satisfaction), (2) l'espace (bienvenue et réseau) et (3) la joie (récompense). Cette considération si elle s'avère serait de pure imagination de la part du sujet énonçant puisqu'en affirmant avoir eu à poser des actes en relation avec la « satisfaction » de sa clientèle, tout ce qu'une banque peut espérer ne peut concourir qu'à sa conjonction avec l'objet valeur qu'est la performance. D'où la récompense énoncée supra.

En fait, il y a une double satisfaction: celle du client qui implique et entraine celle de la banque par le truchement de la proximité ainsi créée. La satisfaction de la banque n'est possible que si et seulement si le client est satisfait. En d'autres termes, la satisfaction du client a une double plus-value performative à savoir que la banque est récompensée au propre comme au figuré: une véritable syllepse de sens.

\subsection{Posts de Société Générale Cameroun: de l'ontique à l'aléthique}

Sur le plan scripto-esthétique, et à quelques limites près, les posts de Société Générale Cameroun ont la même configuration que ceux de SCB Cameroun. En effet, le premier post (Image 3) est un bandeau monté grâce à l'infographie avec un accent sur le service digital (le jeune homme de 
l'affiche est dans une situation d'appel téléphonique); et le deuxième post (Image 4) est prise en situation réelle devant une agence montrant un couple de clients se servant dans un distributeur automatique installé à l'extérieur.

Image 3 - Capture d'écran du post de la photo de couverture de la page facebook de Société Générale Cameroun

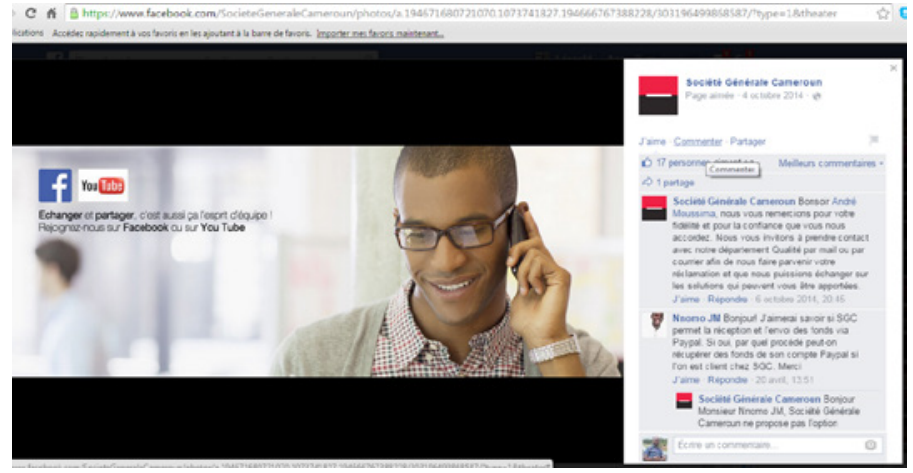

Source: Page Facebook de Société Générale Cameroun

Image 4 - Capture d'écran relatif à l'ouverture d'une agence de Société Générale Cameroun

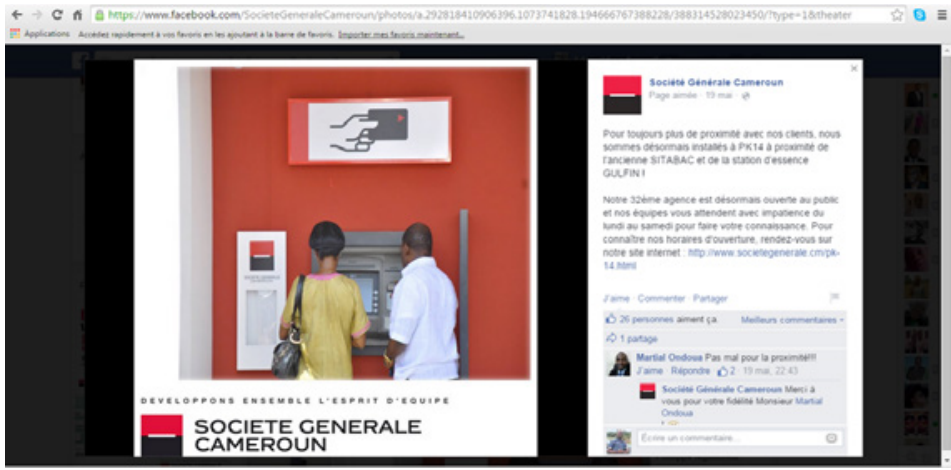

Source: Page Facebook de Société Générale Cameroun 
Sur le premier post illustratif de la photo de couverture de Société Générale Cameroun (Image 3), l’on voit un jeune homme en lunettes en train de recevoir un appel téléphonique. Vêtu d'une chemise carrelée (rouge, blanc bleu), couverte d'un pull-over, une montre en diamant à la main gauche qui tient le téléphone à l'oreille et d'un sourire discret, ce jeune homme, la trentaine révolue, est le prototype d'un client moderne de statut-médian de par sa vêtue et sa tenue posturo-kinésique.

Sur le fond du bandeau, l'on voit un fond plastique d'un blanc flouté avec le logo de Facebook et celui de YouTube (avec Twitter et LinkedIn, ces deux médias sociaux font partie des quatre les plus utilisés au Cameroun). Le texte de signature, juste en dessous des deux logos est celui-ci: «Échanger et partager, c'est aussi ça l'esprit d'équipe ! Rejoignez-nous sur Facebook ou sur YouTube».

Sous l'angle de l'implicite, l'on peut lire dans ce discours une conformité de la banque aux exigences de la modernité notamment la banque 2.0. Cette modernité est validée dans le discours de conseil et d'exhortation (acte promissif): «rejoignez-nous», «Échanger et partager...». Comme SCB Cameroun, Société Générale Cameroun est dans un individualisme personnifiant. Cet individualisme peut alors se décliner ainsi : «je suis...», «c'est permis... de nous suivre sur (dans) ...»: de la modalité ontique (l'être) à la modalité déontique (le devoir). Cette transformation du discours relationnel de Société Générale Cameroun de l'ontique au déontique est, sur le plan commercial, prudent et stratégique. En procédant par un «acte directif » qui, selon Searle (1982), amène l'interlocuteur à réagir, à faire quelque chose à la suite de l'acte illocutoire du destinateur, l'énonciateur Société Générale Cameroun ne se glorifie pas comme le fait SCB Cameroun dans ses posts, mais laisse l'opportunité à 
l'internaute de découvrir par lui-même son univers.

À juste titre, cette posture énonciative est en harmonie avec la signature de cette marque bancaire, à savoir: «développons ensemble l'esprit d'équipe». En fait, il est de notoriété publique que dans une équipe, on n'impose pas, plutôt on partage et on échange.

Sur son deuxième post (Image 4), Société Générale Cameroun annonce l'ouverture d'une nouvelle agence dans le quartier PK14 de la ville de Douala (capitale économique du pays). De dos, on y voit un couple (un homme en chemise blanche et une femme en boubou vert-jaunâtre avec un sac noir sur son bras gauche) utilisant en commun le distributeur magnétique aux couleurs de l'entreprise avec une signature de l'entreprise placée en baseline lors du montage du bandeau.

Le texte qui accompagne ce post est le suivant:

Pour toujours plus de proximité avec nos clients, nous sommes désormais installés à PK14 à proximité de l'ancienne SITABAC et de la station d'essence GULFIN ! Notre $32^{\text {ème }}$ agence est désormais ouverte au public et nos équipes vous attendent avec impatience du lundi au samedi pour faire votre connaissance. Pour connaître nos horaires d'ouverture, rendez-vous sur notre site internet : http://www. (lire: site web de la banque)

Comme dans le post de sa photo de couverture, le sujet énonçant Société Générale Cameroun opte pour un discours de promesse et d'explication, c'est-à-dire respectivement un " acte promissif » et un " acte expositif », au sens de Austin (1970, p. 113). Ce procédé énonciatif met en valeur l'individualisme du sujet parlant («nous sommes», «notre $32^{\text {ème }}$ ", «nos équipes») dans une modalité ontique vers des modalités déontiques sous la forme implicite suivante: «je 
suis...», «Je me permets de... de nous rejoindre... où nos équipes vous attendent...». Une fois de plus, le sujet énonçant Société Générale Cameroun préfère l'humilité, situe et interpelle ses clients et prospects sur un ton «respectueux», et non contraignant, qui laisse le client apprécier les promesses tenues par la banque sur le «lieu de vente».

\subsection{Prise en charge du «discours relationnel» de SCB Cameroun}

Presque tous les médias sociaux ont un certain nombre de points axiologiques qui les unissent : participation, ouverture, conversation, communauté, interconnexion. L'acte de poster un message sur sa page Facebook présuppose automatiquement pour une banque, a priori, l'acceptation de se mettre en relation discursive avec la communauté des facebookers.

C'est ainsi qu'aussitôt mis en ligne, chaque post s'inscrit dans une «rencontre» avec le social. Autrement dit, la «prise en charge» d'un post par un dispositif interactif transforme automatiquement tous les participants à la discussion en coénonciateur du message.

Comme nous l'avons relevé supra, notre analyse des discours relationnels des banques à travers leurs posts sur Facebook relève du regard sociosémiotique au sens de Semprini (2007, p. 13). Ce regard sociosémiotique consistera à inscrire dans une «discursivité sociale» comprise cette fois-là dans son «acception technique d'objets de sens énoncés, ou bien pris en charge parun dispositifénonciatif, ou bien encore, en simplifiant, pris en charge par des sujets sociaux». Dans les paragraphes qui ont précédé, nous avons tenté de montrer comment à travers ses deux posts (Image 1 et 2), SCB Cameroun affirmait 
une modalité ontique vers des modalités épistémiques autour des valeurs telles que la diversité de services, la proximité, la satisfaction, la récompense, la convivialité.

Chronologiquement, voici les quatre (04) premières interactions $^{3}$ avec les internautes sur son premier post (Image 1):

Internaute 1 : mon dossier traine chez vs

Internaute 2 : toi tu as tjrs les dossiers en instance!!!...ton gestionnaire s'occupe...

Internaute 3 : Le boss sa di koi la scb ce bien no?

Internaute 1 : hahahaha, Internaute 1 , on gere les grd dossiers

Puis, celles du deuxième post (Image 2):

Internaute 1a : pourquoi les distributeurs sont-ils toujours aussi exposés??

Internaute 2a : Enfin vraiment, parce que quand on se retrouvait dans ces zones là, l'on se demandait pourquoi on es client SCB Cameroun

Internaute 3a: Boum Mbadi Ati jari wafa

Internaute 4a: embauchez nous meme noh, mes responsables de la DRH

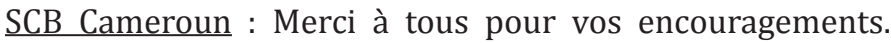
Mbouda et Bafang sont en cours d'ouverture. Meilleurs vœux. Internaute 5a : Satisfaire le client, c'est une bonne chose. Mais j'espère bien que cela passe par son écoute! J'ai pas l'impression que ce soit le cas de toute façon.

Internaute 6a: mais la qualité de vos produit et service laisse a désiré

Internaute 7a : C’est cela la proximité!!!!!!!!!!!!!!!!!!!!!!!!!!!!

3 Nous avons préféré garder l'anonymat des internautes en leur attribuant des noms génériques numérotés suivant l'ordre de leur apparition dans l'échange. 
En considérant que le registre de langue du Web 2.0 est de type texto, on réussit à déchiffrer les textes et le sens à leur donner par l'internaute énonciateur. Quand la SCB Cameroun expose et affiche son cœur de métier, l'on peut y remarquer tantôt un acquiescement, tantôt une remise en question des modalités revendiquées, par les internautes. La réplique de l'Internaute 6a montre bien comment il y a une remise en question du positionnement énonciatif de SCB sur son activité: «mais la qualité de vos produits et services laisse à désirer». Ce désaccord verbal peut se justifier dans la mesure où dans cette «interaction virtuelle», on entend à peu près l'influence réciproque que les participants exercent sur leurs actions respectives (GOFFMAN, 1973, p. 11).

En fait, «le désaccord est susceptible d'impliquer l'exercice du pouvoir, parce qu'il entraîne un "conflit" et ainsi un "affrontement d'intérêts"» (LOCHER, 2004, p. 93), notre traduction $)^{4}$. Dans ce cas précis, la remise en question que porte cet internaute $6 \mathrm{a}$ (en posture d'anti-sujet) relève d'une préoccupation qui montre qu'au-delà de l'affirmation de la satisfaction et de la récompense par le sujet énonciateur SCB Cameroun, il y a bel et bien des problèmes qui persistent et qui relèvent de la qualité du service, caractéristique fondamentale de l'activité bancaire. En effet, ces problèmes apparaissent implicitement dans les réactions des internautes.

Concernant les valeurs mises en exergue par le même sujet énonçant SCB Cameroun, nous avons souligné que le fait de s'affirmer vers des formes modales épistémiques est susceptible de causer préjudice à l'e-réputation de l'entreprise aux yeux des internautes.

4 M. Locher, Power and politeness in action, «disagreement is likely to involve the exercise of power, because it entails a "conflict" and therefore also a "clash of interests"» (LOCHER, 2004, p. 93). 
À titre d'exemple, pour exprimer leur désaccord à l'affirmation de SCB Cameroun, des internautes exposent dans leurs posts le doute et l'indifférence. Ce cas de posture préfigure dans le post de l'Internaute 5a: «Satisfaire le client, c'est une bonne chose. Mais j'espère bien que cela passe par son écoute! J'ai pas l'impression que ce soit le cas de toute façon». Cette réplique de l'internaute s'inscrit dans une nonconfiance vis-à-vis du discours relationnel du sujet énonçant qui a pris le risque de construire sa modalité ontique vers les modalités épistémiques, à savoir le certain et le plausible. Or, sur la même valeur modale, les internautes peuvent choisir de s'y opposer à travers l'incertain et le contestable.

Un autre constat de conflit entre le discours interactif de la banque et les réactions des internautes est la diversion. Ce désaccord qui se lit sous la forme d'une contestation verbale consiste pour les internautes à "désorienter » l'objet de l'échange en créant ainsi une forme de distraction qui laisse voir dans les interactions des préoccupations essentiellement personnelles de la part des internautes et qui n'ont aucune relation avec le sujet en discussion dans le post original.

C'est dans cette même optique que s'inscrit les posts respectifs des Internautes 1: mon dossier traine chez vs (lire: mon dossier traine chez vous); 3: Le boss sa di koi la scb ce bien no? (lire: le boss, comment vas-tu? Le travail marche à la SCB n'est-ce pas?); et celui de l'internaute 4a: «embauchez nous meme noh» (lire: embauchez nous donc! Qu'y a-t-il même?).

Cependant, il est à considérer qu'en contexte, si des interventions peuvent s'inscrire dans une logique de diversion, chacun des internautes peut tout aussi poser un problème spécifique non moins pertinent. C'est ainsi qu'on peut sousentendre que l'Internaute 1 exprime implicitement dans son 
post la lenteur dans le traitement des dossiers; l'Internaute 3 dans son expression connaîtrait l'Internaute 1 qu'il appelle «boss» afin de bénéficier d'une attention particulière extrabancaire avec celui-ci et qui relève du pathos exprimé par cet Internaute 3. Le terme «boss» concourt effectivement de ce pathos.

Par contre, l'internaute 4 a quant à lui esquive l'objet du débat pour solliciter son recrutement comme employé au sein de la banque. Il est donc hors sujet et vraisemblablement en situation de chômage. L'occasion que lui offre Facebook (comme les autres réseaux sociaux) lui permet de poser un problème à un moment inopportun et au mauvais endroit.

Comme on vient de le constater, la prise de décision d'un établissement de crédit, ou de toute autre entreprise, de s'ouvrir aux «nouveaux médias» présente aussi bien des inconvénients (risques) que des avantages et opportunités. Pour ce qui est des risques mal gérés, ils peuvent porter atteinte non seulement à l'image de l'entreprise mais également à sa e-réputation si le flux conversationnel qu'entraine ses posts n'est pas géré avec célérité, souplesse, prudence et pertinence. Dès cet instant, le problème de préservation de face au sens de Gollfman (1973 [1959], p. 21-22) surgit:

Interagir avec l'autre représente un double risque, celui de donner une image négative de soi et celui d'envoyer à l'autre une image négative de lui-même. Tout discours est construit en tenant compte de cette double contrainte et contient des techniques défensives (defensive practices) émises pour protéger ses propres projections et des techniques de protection (protective practices) émises pour sauvegarder la définition de la situation projetée par les autres.

C'est en effet dans une nécessité de préserver sa face que 
SCB Cameroun dans son deuxième post (Image 2) intervient pour répondre avec une certaine politesse aux préoccupations des uns et des autres et surtout pour atténuer les conflits nés des interactions avec les internautes qui sollicitent chacun, une solution à ses problèmes et inquiétudes, ici et maintenant. Pour répondre à toutes les préoccupations, le co-énonciateur SCB Cameroun réagit en ces termes : «Merci à tous pour vos encouragements. Mbouda et Bafang sont en cours d'ouverture. Meilleurs vœux».

En pratique, ce rôle de modération au co-énonciateur n'est en rien qu'une visée stratégique de «régulation», mieux, de préservation de la face au risque de laisser les internautes dévier l'objet des échanges et de créer ainsi de nouveaux débats n'ayant aucune relation directe avec le post original.

Or, généralement dans ces cas de figure, comme le community manager de SCB Cameroun semble ne pas s'être approprié des outils et techniques de modération et de relance des discussions, les objectifs de quête et de rétention des clients qui viseraient l'entreprise à travers ses prises de parole sur Facebook, y restent sans solutions véritables. En fait, en remerciant les internautes et en leur annonçant l'ouverture d'autres agences (dans les villes de Mbouda et de Bafang), le community manager ne répond véritablement à aucune des préoccupations exprimées par les internautes par le fait de la liberté d'expression que leur offrent les réseaux sociaux. Il y a également là un problème de qualité de service si le community manager évite de répondre précisément aux problèmes soulevés par les internautes même si ceux-ci ne sont pas toujours en adéquation avec le post initial. D'où le sentiment de la non-maîtrise de la gestion de ce «nouveau média» pourtant essentiel qu'est Facebook par cet actant dans la gestion des posts. 


\subsection{Prise en charge du «discours relationnel» de Société Générale Cameroun}

Comme dans le cas de SCB Cameroun, les posts de Société Générale Cameroun sont mis en discursivité à travers les échanges qui les inscrivent également dans un conflit sous la forme de désaccord verbal.

De manière chronologique, voici les interactions des internautes sur son premier post (Image 3):

Internaute $1 \mathrm{~b}$ : ma banque qui me fait vivre 2 puis btôt + de 20 ans, mais je 8 suis déçu par sa prise 2 décision à mon égard et je veux le signifier à sa direction. Sinon je me sens bien ds cette banque

Société Générale Cameroun : Bonsoir Internaute $1 \mathrm{~b}$, nous vous remercions pour votre fidélité et pour la confiance que vous nous accordez. Nous vous invitons à prendre contact avec notre département Qualité par mail ou par courrier afin de nous faire parvenir votre réclamation et que nous puissions échanger sur les solutions qui peuvent vous être apportées.

Internaute 2b : Bonjour! J'aimerai savoir si SGC permet la réception et l'envoi des fonds via Paypal. Si oui, par quel procédé peut-on récupérer des fonds de son compte Paypal si l'on est client chez SGC. Merci

Société Générale Cameroun : Bonjour Monsieur Internaute 2b, SG Cameroun ne propose pas l'option Paypal mais avec nos cartes bancaires Visa, vous pourrez effectuer vos achats en toute sécurité sur Internet. Pour en savoir plus, nous vous invitons à consulter notre offre présentée sur (lire : le site web de la banque) (Rubrique Particuliers > Nos cartes) Excellente journée.

Internaute $3 \mathrm{~b}$ : www.(un site web commercial) ${ }^{5}$

5 Pour des raisons de type publicitaire, nous avons opté de remplacer les sites web par le texte «site web de ...». 
Semblablement, les échanges du deuxième post (Image 4) se présentent ainsi qu'il suit :

Internaute 1c: Pas mal pour la proximité!!!

Société Générale Cameroun : Merci à vous pour votre fidélité Monsieur Internaute 1c :

Internaute 2c : avec vos distributeurs qui sont toujours HS. Société Génarale Cameroun : Bonjour Monsieur, SG Cameroun vous présente ses excuses pour la gêne occasionnée. Nos équipes sont à pied d'œuvre pour rétablir chaque distributeur défectueux de notre réseau afin d'offrir la meilleure qualité de service à nos clients. Pourriez-vous nous indiquer où se situe le distributeur défectueux afin que nos équipes puissent intervenir au plus vite? Nous vous remercions par avance et restons à votre disposition pour répondre à vos questions.

Internaute 3c: Surtout SGC New Deido pas la peine. On ne peut pas aller là bas n'importe comment.

Internaute 4c: SGBC lors de l'ouverture de la prochaine Agence pensez à ma candidature....

Pour prendre le cas de la réclamation de la proximité avec ses clients, lorsque la banque ouvre de nouvelles agences, l'on peut observer une relative unanimité dans les réactions des internautes qui expriment des idées en conjonction avec le post original du sujet énonçant. Les réactions respectives des Internautes $1 \mathrm{~b}$ et $1 \mathrm{c}$ : «ma banque qui me fait vivre 2 puis btôt + de 20 ans, (...) Sinon je me sens bien ds cette banque. (Lire: Ma banque depuis bientôt plus de 20 ans (...) Sinon, je me sens bien dans cette banque)» et «Pas mal pour la proximité!!!», fait vivre des preuves de cette conjonction discursive entre les internautes et le discours de la banque.

Cette attitude serait due à la « démarcation discursive » du sujet énonçant Société Générale Cameroun qui s'attèle 
à produire un individualisme qui laisse percevoir une modalité ontique vers des modalités aléthiques avec pour particularité de mobiliser des actes promissifs et expositifs qui donnent l'occasion aux énonciataires de vérifier ses dires sur le lieu de vente.

Cependant, en ce qui concerne les propositions en relation avec la qualité de service qui est implicite dans le discours de Société Générale Cameroun, la plupart des internautes ne valident pas la proposition du sujet énonçant et deviennent des anti-sujets. Dans ces cas, l'on peut percevoir plusieurs conflits entrainant des désaccords verbaux. En effet, l'on relève dans les propos et réponses des internautes, tantôt l'infirmation et la méconnaissance, tantôt la remise en question des propositions énoncées par la banque notamment l'interactivité qui est sous-entendue par l'échange téléphonique du personnage du bandeau de la photo de couverture de Société Générale Cameroun.

C'est ainsi que l'on peut lire:

Internaute $3 \mathrm{~b}: \mathrm{www}$.(un site web commercial)

Internaute 2c : avec vos distributeurs qui sont toujours HS. (Lire : Vos distributeurs automatiques sont toujours « hors sujet », c'est-à-dire non-fonctionnels)

Internaute 3c: Surtout SGC New Deido pas la peine. On ne peut pas aller là bas n'importe comment. (Lire : l'agence SG Cameroun de New Deido ne vaut pas la peine. L'insécurité y règne parce que les distributeurs automatiques sont ouverts même à la vue des personnes malintentionnées).

Dans leurs désaccords verbaux, ces répliques des internautes posent parallèlement des problèmes qui remettent en cause le discours relationnel que construit la banque. Ces problèmes dans leur ensemble ont trait à la 
manipulation et aux propos hors-cadre (cas de l'Internaute 3b); à l'insécurité liée aux distributeurs automatiques installés à l'extérieur lesquels exposent les clients dans une insécurité permanente lors des opérations de retrait d'argent (cas de l'Internaute 2c); à la mauvaise qualité de service qu'offrent les distributeurs automatiques, notamment leur lenteur et leurs états défectueux (cas de l'Internaute 3c, etc.

C'est donc fort heureusement que contrairement à la SCB Cameroun dans le rôle de co-énonciateur, Société Générale Cameroun essaye de sauver sa face grâce à son instantanéité et l'attention qu'elle prend pour répondre à chaque préoccupation des internautes de manière personnalisée.

Les extraits suivants mettent en lumière ces différentes «interventions régulatrices» de Société Générale Cameroun dans ses réactions et interactions avec les internautes:

Dans le post de sa photo de couverture (Image 3), le community manager de Société Générale Cameroun réagit en deux temps pour apporter des réponses aux commentaires des internautes, et également les rassurer par la même occasion:

Société Générale Cameroun (Image 3, Temps 1): Bonsoir Internaute $1 \mathrm{~b}$, nous vous remercions pour votre fidélité et pour la confiance que vous nous accordez. Nous vous invitons à prendre contact avec notre département Qualité par mail ou par courrier afin de nous faire parvenir votre réclamation et que nous puissions échanger sur les solutions qui peuvent vous être apportées.

Société Générale Cameroun (Image 3, Temps 2) : Bonjour Monsieur Internaute $2 \mathrm{~b}$, SG Cameroun ne propose pas l'option Paypal mais avec nos cartes bancaires Visa, vous pourrez effectuer vos achats en toute sécurité sur Internet. Pour en savoir plus, nous vous invitons à consulter notre offre présentée sur (lire : le site web de la banque) (Rubrique Particuliers > Nos cartes) Excellente journée. 
Société Générale Cameroun (Image 4) : Bonjour Monsieur, SG Cameroun vous présente ses excuses pour la gêne occasionnée. Nos équipes sont à pied d'œuvre pour rétablir chaque distributeur défectueux de notre réseau afin d'offrir la meilleure qualité de service à nos clients. Pourriez-vous nous indiquer où se situe le distributeur défectueux afin que nos équipes puissent intervenir au plus vite? Nous vous remercions par avance et restons à votre disposition pour répondre à vos questions

À l'observation, les posts-réponses du community manager de la page Facebook de Société Générale Cameroun semblent bien mesurer les caractéristiques et fonctions des médias sociaux au service du marketing relationnel 2.0 des banques.

Comme Balagué et Fayon (2012, p. 12), on reconnaît aujourd'hui que les réseaux sociaux numériques constituent une opportunité pour les entreprises et les marques bancaires à condition que ces dernières réussissent à s'approprier les paradigmes qui les caractérisent en termes de stratégies d'entreprise par rapport aux médias traditionnels comme la télévision, la radio, la presse écrite, le cinéma, le livre:

[Avec les médias sociaux], l'entreprise devra aussi de plus en plus appliquer le concept de coproduction de valeur en intégrant le consommateur comme acteur dans sa stratégie : ce consommateur pourra participer aux innovations, aider l'entreprise à construire des bases de données (méls d'amis), devenir vendeur pour l'entreprise, noter des marques, diffuser des informations sur les produits, viraliser des films publicitaires. Les réseaux sociaux constituent des outils efficients pour développer une telle stratégie, mais cela suppose aussi que l'entreprise rentre dans la netiquette des réseaux, à savoir la transparence, l'écoute, la conversation, l'interaction. 
Plus clairement, on a le sentiment que Société Générale Cameroun dans ses prises de parole essaye de résoudre plusieurs problèmes à la fois. Ainsi, les prises de parole de Société Générale Cameroun semblent être sous-tendues par un individualisme qui se conjugue à la troisième personne du singulier et à la première personne du pluriel, à travers une stratégie de «nous exclusif» qui a un seul objectif : présenter l'entreprise comme un partenaire qui reconnaît ses manquements techniques ainsi que les préjudices causés à ses clients, mais également montrer une entreprise qui s'engage à les corriger et à les améliorer. Les exemples suivants constituent de cette démarche relationnelle et communicationnelle de Société Générale Cameroun: «... nous vous remercions pour votre fidélité et pour la confiance», « Nous vous invitons à prendre contact avec ...», «Bonjour Monsieur Internaute $2 \mathrm{~b}$, Société Générale Cameroun ne propose pas...», «... Pour en savoir plus, nous vous invitons à ...».

Cette démarcation discursive que doit jouer un community manager est d'autant plus importante qu'en tant que plateforme fondée sur l'instantanéité, l'interactivité, la personnalisation, l'écoute, le partage ..., Facebook est un espace communautaire qui regroupe des utilisateurs aux profils pluriels dont les activités et la présence obéissent aux enjeux divers et parfois opposés. Pour ce faire, essayer d'écouter, de suivre, d'informer et surtout d'«engager» les membres de sa communauté facebook en faveur de son e-réputation constitue des atouts incommensurables pour bâtir la confiance et conforter sa performance à travers un discours relationnel savamment entretenu. 


\section{En guise de conclusion}

Partant de l'idée que Facebook et les autres réseaux ont fondamentalementmodifiélespratiquescommunicationnelles et commerciales des entreprises en direction de leurs publics en termes de conquête, de maintien et de rétention des prospects et clients, l'on peut également reconnaître que sur le plan paradigmatique, l'on est ainsi passé de la société de l'information à la société de la recommandation. De nos jours, comme James Surowiecki, on est tenté de croire à une triple fonction qu'assureraient les réseaux sociaux pour les entreprises:

Les réseaux sociétaux intègrent la cognition, la coordination et la coopération. La cognition pour trouver la réponse à une question complexe. La coordination pour agir en fonction du comportement des autres. La coopération pour se « réguler » avec l'action des autres. Ainsi est né le crowdsourcing : l'usage de groupes interreliés pour répondre collectivement à des questions difficiles nécessitant un avis d'experts (SUROWIECKI, 2008, p. 10-11).

Les discours relationnels de SCB Cameroun et Société Générale Cameroun, pris comme des sujets énonçants et devenant de fait des co-énonciateurs sur leur propre page Facebook, nous a permis de valider que ce réseau social numérique est un espace d'expressions de l'individualisme par excellence pour ses utilisateurs. En tant qu'individus SCB Cameroun y construit un discours fondé sur une modalité ontique vers des modalités épistémiques alors que Société Générale Cameroun met plutôt en jeu une modalité ontique orientée vers des modalités aléthiques. Si ces deux approches 
discursives constituentle fil conducteur des deux banques dans leur communauté Facebook, c'est également à partir d'elles que chacune des banques réussit à organiser son marketing relationnel 2.0 face aux désaccords verbaux marqués sur les posts de SCB Cameroun, relativement maîtrisés dans le cas de Société Générale Cameroun.

Par ailleurs, l'analyse sociosémiotique a ainsi permis de confronter les thèmes centraux mis en jeu par chacune des banques en termes de confiance, de proximité, de satisfaction de la clientèle, de la qualité du service, de partage d'un certain nombre de valeurs avec les internautes qui par principe sont également des co-énonciateurs des messages dans le jeu des interactions et de prise en charge des posts à visées stratégiques des banques considérées.

En fin de compte, au moins trois observations techniques et stratégiques ont constitué à nos yeux des obstacles à lever par les marques bancaires en contexte camerounais dans leur quête de performance: (1) faible taux d'engagement sur les pages Facebook des banques objet de l'étude; (2) maîtrise approximative des techniques et des approches particularisantes des réseaux sociaux qui se présentent pourtant comme un outil de marketing relationnel pertinent de conquête, de recrutement et de rétention des clients; (3) les choix approximatifs des positionnements discursifs des messages textuels qui accompagnent la publication des posts en termes de visées stratégiques spécifiques en fonction des différentes campagnes promotionnelles et commerciales des banques dans leur ensemble.

Dès lors, si au double plan stratégique et narratif, les " modalités de faire » des établissements de crédit dans leurs différentes instances d'échange relationnel mettent 
en scène un «sujet» en posture de quête d'un objet valeur (la performance par exemple), il est fondé de croire que les médias sociaux constituent à n'en point douter des adjuvants non moins importants de cette quête. L'autre problème étant de savoir à partir de quel moment peut-on parler de performance dans le cas des établissements bancaires à partir des réseaux sociaux. Cette préoccupation a été résolue par des recherches en sciences de gestion. Elle pose qu'en effet, la performance d'un établissement bancaire ne peut pas être de l'exclusivité des indicateurs comptables et financiers mais également des variables non-comptables de type qualitatif et intangible à l'instar de la qualité de la relation client qui sousentend la confiance comme valeur prééminente de l'activité bancaire.

\section{RÉFÉRENCES}

AUSTIN, J. Quand dire c'est faire. Paris: Le Seuil, 1970.

BALAGUÉ, C.; FAYON, D. Facebook, Twitter et les autres... Intégrer les réseaux sociaux dans une stratégie d'entreprise. Paris: Pearson, 2012.

\section{BALLY, C. Linguistique générale et linguistique française.} Berne: A. Francke AG Verlag, [1932]1965.

BERTHON, P. R. et al. Marketing meets Web 2.0, social media and creative consumers: implications for international marketing strategy. Business Horizons, v. 55, p. 261- 271, 2012. 
BOTTON, D. Au commencement était le nom..., (interview). In: GILLES, G. (éd.). Horizons bancaires, Paris, n. 325, juin, p. 35-40, 2005.

CharaUdeau, P.; MAInguenEAU, D. (ÉDs.). Dictionnaire d'analyse du discours. Paris: Seuil, 2002.

FONTANILLE, J. Sémiotique du discours. Limoges: PULIM, 1998.

GANGULI, S. et al. Relationship marketing: a critical evaluation of research streams, ASBBS Annual Conference, Las Vegas: v. 16, n.1, febr. p. 3-6, 2009.

GOFFMAN, E. La mise en scène de la vie quotidienne, t. 1: La présentation de soi. Paris: Minuit, 1973. Trad. fr. de The Presentation of Self in Everyday Life, New-York: Doubleday Anchor, [1959].

GOSSELIN, L. Relations temporelles et modales dans le "conditionnel journalistique". In: DENDALE, P.; TASMOWSKI, L. (ÉDs). Le conditionnel en français. Paris: Klincksieck, 2001. p. $45-66$

KAPFERER J.-N. Les banques sont-elles des marques? In: GILLES, G. (Éd.). Horizons bancaires, Paris, n. 325, juin, p. 25-34, 2005.

LANDOWSKI, E. La société réfléchie. Paris: Seuil, 1989.

LEWI, G. Mythologie des marques: quand les marques font leur storytelling. Paris: Village Mondial-Pearson Education France, 2009. 
LOCHER, M.A. Power and politeness in action. Berlin: Mouton de Gruyter, 2004.

MORIN, E. M. et al. L'Efficacité de l'organisation. Théories, représentations et mesures. Montréal: Gaëtan Morin, 1994.

PESQUIEU, Y. La notion de performance globale en question. $5^{\text {EME }}$ FORUM INTERNATIONAL ETHICS. Tunis, 1-2 déc., 2004.

REBILLARD, F. Du Web 2.0 au Web 2: fortunes et infortunes des discours d'accompagnement des réseaux socionumériques. Hermès, n. 59, p. 25-30, 2011.

SEARLE, J. R. Sens et expression. Étude des théories des actes de langage. Trad. Joëlle Proust. Paris: Minuit, 1982.

SEMPRINI, A. (ÉD.). Analyser la communication 2. Comment analyser la communication dans son contexte socioculturel. Paris: L'Harmattan, 2007.

SERRES, M. Petite Poucette. Paris : Éditions Le Pommier, 2012.

SUROWIECKI, J. La Sagesse des foules. Paris: Éditions JeanClaude Lattès, 2008.

WALDRON, V. R.; APPLEGATE J. L. Interpersonal construct differentiation and controversial planning: an examination of two cognitive accounts for the production of competent verbal disagreement tactics. In: Human communication research. n. 21, p. 3-35, 1994. 
Léopold NGODJI TCHEUTOU, Alphonse Joseph TONYÈ

Artigo recebido em setembro de 2015 e aprovado em dezembro de 2015.

Disponível em: http://seer.fclar.unesp.br/casa 